\title{
Charm hadronic physics at Belle
}

\author{
Alexey Drutskoy*† \\ University of Cincinnati, USA \\ E-mail: drutskoi@bmail.kek.jp
}

We report an improved measurement of $\bar{B}^{0} \rightarrow D_{s J}^{+} K^{-}$decays using a data sample of $357 \mathrm{fb}^{-1}$ and studies of $D_{s 1}(2536)^{+} \rightarrow D^{+} \pi^{-} K^{+}, D_{s 1}(2536)^{+} \rightarrow D^{*+} K_{S}^{0}$ and $D^{0} \rightarrow K^{-}\left(\pi^{-}\right) l^{+} v$ decays using a data sample of $282 \mathrm{fb}^{-1}$. The data used in these analyses were collected by the Belle detector at the KEKB asymmetric energy $e^{+} e^{-}$collider. We measure $\mathscr{B}\left(\bar{B}^{0} \rightarrow D_{s J}^{*}(2317)^{+} K^{-}\right) \times \mathscr{B}\left(D_{s J}^{*}(2317)^{+} \rightarrow D_{s}^{+} \pi^{0}\right)=(4.4 \pm 0.8 \pm 0.6 \pm 1.1) \times 10^{-5}$ and $\mathscr{B}\left(\bar{B}^{0} \rightarrow D_{s J}(2460)^{+} K^{-}\right) \times \mathscr{B}\left(D_{s J}(2460)^{+} \rightarrow D_{s}^{+} \gamma\right)=\left(0.53 \pm 0.20_{-0.15}^{+0.16}\right) \times 10^{-5}$. The decay $D_{s 1}(2536)^{+} \rightarrow D^{+} \pi^{-} K^{+}$is observed for the first time. An angular analysis of the $D_{s 1}(2536)^{+} \rightarrow$ $D^{*+} K_{S}^{0}$ decay is performed. Semileptonic $D^{0}$ decays are studied using a global reconstruction method that provides very good resolution in neutrino momentum and the momentum transfer $q^{2}$.

International Europhysics Conference on High Energy Physics

July 21st - 27th 2005

Lisboa, Portugal

* Speaker.

† On behalf of the Belle Collaboration 


\section{Improved measurements of $\bar{B}^{0} \rightarrow D_{S J}^{+} K^{-}$decays}

An updated study of the decays $\bar{B}^{0} \rightarrow D_{s J}^{+} K^{-}$[1] was performed with a data sample that is approximately 2.5 times larger than in the paper published recently by Belle [2] that first reported the $\bar{B}^{0} \rightarrow D_{S J}^{*}(2317)^{+} K^{-}$decay mode. The $\bar{B}^{0} \rightarrow D_{s(J)}^{+} K^{-}$decays can be described by a PQCD factorization $W$ exchange process $[3,4]$ or, alternatively, by final state interactions [5, 6]. Assuming there is a four-quark component of the $D_{s J}$ mesons, the tree diagram with $s \bar{s}$ pair creation may also contribute [2].

In this analysis we applied the same selection criteria as in [2], where a detailed description of the criteria can be found. The $\Delta E=E_{B}^{C M}-E_{\text {beam }}^{C M}$ and $\Delta M\left(D_{s J}\right)=M\left(D_{s J}^{+}\right)-M\left(D_{s}^{+}\right)$distributions for the $D_{s J}^{+} K^{-}$combinations are shown in Fig. 1 for the range $5.272 \mathrm{GeV} / \mathrm{c}^{2}<M_{\mathrm{bc}}<5.288 \mathrm{GeV} / \mathrm{c}^{2}$, where $E_{B}^{C M}$ and $E_{\text {beam }}^{C M}$ are the $B$ candidate and beam energies in the center-of-mass system. The $\Delta E$ distributions are modeled using a linear background function and a Gaussian signal shape (the Crystal Ball shape function [7] is used for the $D_{S J}(2460)^{+}$) with zero mean and a fixed width determined from MC data. The $\Delta M\left(D_{S J}\right)$ distributions are described by the sum of a signal Gaussian and a linear background. The widths of the Gaussians are fixed from MC while the peak positions are allowed to float. A strong $\bar{B}^{0} \rightarrow D_{s J}^{*}(2317)^{+} K^{-}$signal is observed and evidence of the $\bar{B}^{0} \rightarrow D_{s J}(2460)^{+} K^{-}$signal is also seen.
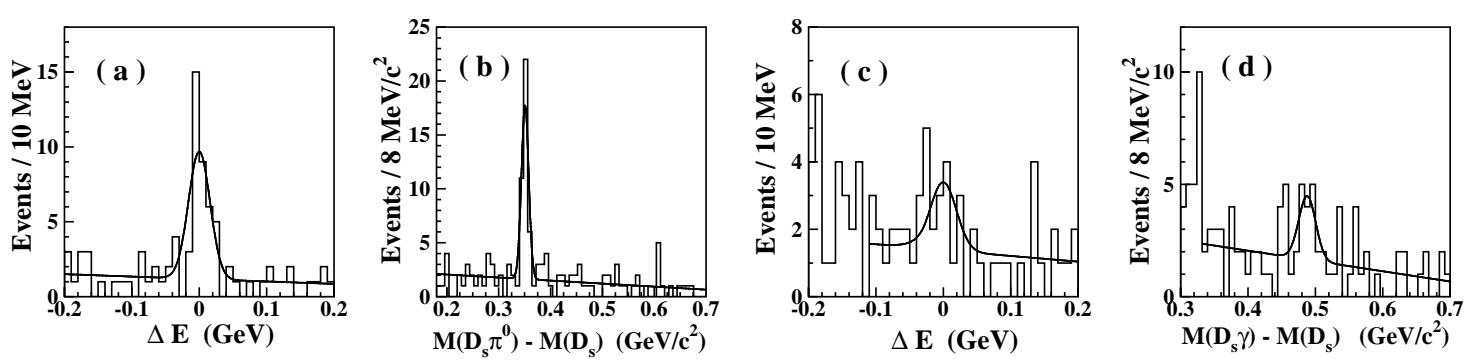

Figure 1: $\Delta E$ (a) and $\Delta M\left(D_{s J}\right)$ (b) distributions for the $\bar{B}^{0} \rightarrow D_{s J}^{*}(2317)^{+} K^{-}$decay, and $\Delta E$ (c) and $\Delta M\left(D_{s J}\right)$ (d) distributions for the $\bar{B}^{0} \rightarrow D_{s J}(2460)^{+} K^{-}$decay.

Signal yields, efficiencies, branching fractions and significances for the studied decay channels are shown in Table 1. The signal yields are obtained from the fits of histograms shown in Fig. 1, where the three $D_{s}^{+}$decay channels $\left(\phi \pi^{+}, K^{* 0} K^{+}\right.$and $\left.K_{S}^{0} K^{+}\right)$are combined.

Results are in a good agreement with, and more sensitive than, the previous measurement [2]. The value of $\mathscr{B}\left(\bar{B}^{0} \rightarrow D_{s J}^{*}(2317)^{+} K^{-}\right)$is of the same order of magnitude as $\mathscr{B}\left(\bar{B}^{0} \rightarrow D_{s}^{+} K^{-}\right)$and significantly larger than the $\bar{B}^{0} \rightarrow D_{s J}(2460)^{+} K^{-}$branching fraction. The experimental results disagree with the naïve expectation [8] that the ratio $\mathscr{B}\left(\bar{B}^{0} \rightarrow D_{s}^{+} h^{-}\right) / \mathscr{B}\left(\bar{B}^{0} \rightarrow D_{s J}^{+} h^{-}\right)$should be similar for $h^{-}=\pi^{-}, K^{-}$or $D^{-}$.

\section{Measurements of $D_{s 1}(2536)^{+} \rightarrow D^{+} \pi^{-} K^{+}$and $D_{s 1}(2536)^{+} \rightarrow D^{*+} K_{S}^{0}$ decays}

The $D_{s 1}(2536)^{+}$resonance was observed in two-body $D^{*} K$ final states many years ago. In this analysis [9] the decay channel $D_{s 1}(2536)^{+} \rightarrow D^{+} \pi^{-} K^{+}$is studied for the first time. Fig. 2 shows the mass spectra for $D^{+} \pi^{-} K^{+}$(left top) and $D^{*+} K_{S}^{0}$ (left bottom) decay modes. Large 


\begin{tabular}{l||c|c|c|c|c}
\hline \hline Decay mode & Yield & Yield & Efficiency & Product $\mathscr{B}\left(\bar{B}^{0} \rightarrow D_{s J}^{+} K^{-}\right) \times$ & Signif. \\
& $\Delta M\left(D_{s J}\right)$ & $\Delta E$ & $\left(10^{-4}\right)$ & $\mathscr{B}\left(D_{s J} \rightarrow D_{s} \pi^{0}(\gamma)\right)\left(10^{-5}\right)$ & $\sigma$ \\
\hline$D_{S J}^{*}(2317)^{+} K^{-}$ & $35.3 \pm 6.4$ & $34.1 \pm 6.6$ & $21.9 \pm 0.6$ & $4.4 \pm 0.8 \pm 0.6 \pm 1.1$ & 9.2 \\
$D_{S J}(2460)^{+} K^{-}$ & $11.2 \pm 5.4$ & $10.2 \pm 5.4$ & $59.5 \pm 1.4$ & $0.53 \pm 0.20_{-0.15}^{+0.16}$ & 3.1 \\
& & & & $<0.86(90 \%$ C.L. $)$ & \\
\hline \hline
\end{tabular}

Table 1: Signal yields, efficiencies, product branching fractions, and significances for the $\bar{B}^{0} \rightarrow D_{S J}^{+} K^{-}$ processes. The first error is the statistical uncertainty, the second is the systematic uncertainty. For the $\bar{B}^{0} \rightarrow D_{S J}^{*}(2317)^{+} K^{-}$decay the uncertainty due to $D_{s}^{+}$decay branching fractions is shown separately as the third error. Product branching fractions are obtained from simultaneous $\Delta M\left(D_{S J}\right)$ fits of three $D_{s}$ decay modes as described in the text.

signals are seen in both modes. The ratio of branching fractions $\mathscr{B}\left(D_{s 1}(2536)^{+} \rightarrow D^{+} \pi^{-} K^{+}\right) /$ $\mathscr{B}\left(D_{s 1}(2536)^{+} \rightarrow D^{*+} K_{S}^{0}\right)$ is measured to be $(2.8 \pm 0.2 \pm 0.4) \%$. The study of two-body invariant masses for the $D^{+} \pi^{-} K^{+}$final state was performed and no clear resonant substructure is found.
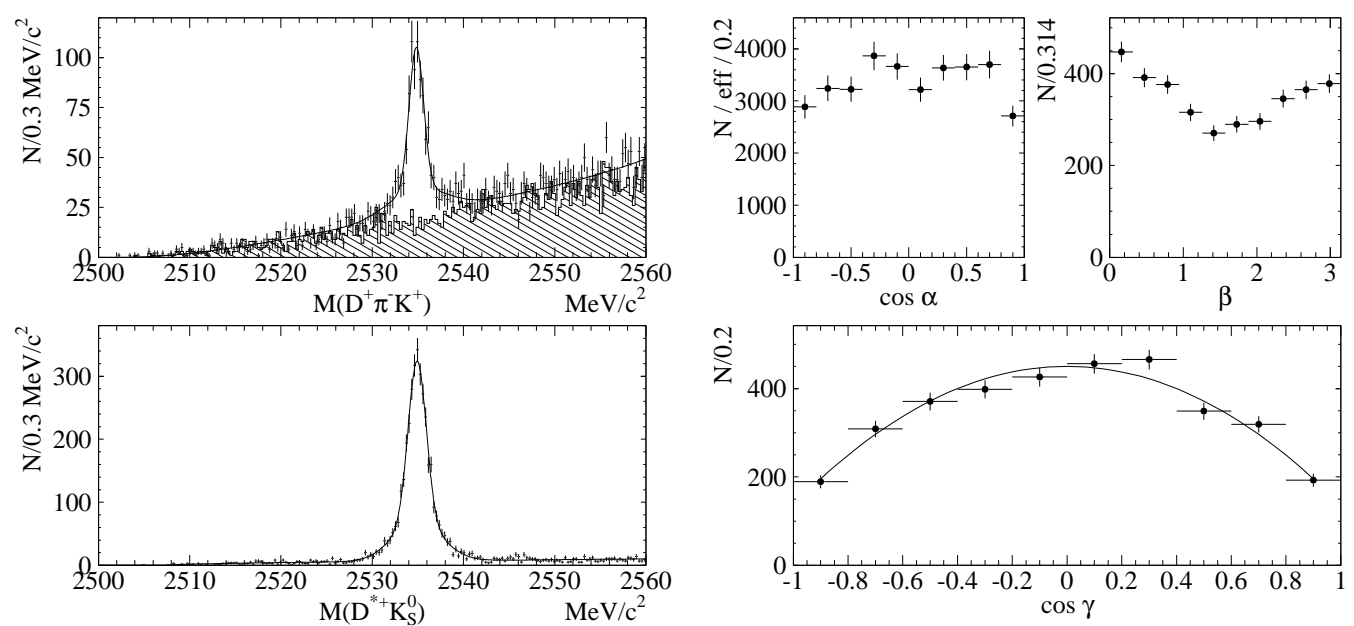

Figure 2: $D_{s 1}(2536)^{+}$mass spectra for $D^{+} \pi^{-} K^{+}$(left top) and $D^{*+} K_{S}^{0}$ (left bottom) decay modes. The hatched histogram in the left top plot shows the corresponding spectrum of wrong sign $D^{+} \pi^{+} K^{-}$combinations. The plots on the right side show angular distributions for the $D_{s 1}(2536)^{+} \rightarrow D^{*+} K_{S}^{0}$ decay. The definitions of angles and the fitting function are described in the text.

An angular analysis of the decay $D_{s 1}(2536)^{+} \rightarrow D^{*+} K_{S}^{0}$ is also performed using the method described in $[10,11]$. The angles $\alpha$ and $\beta$ are measured in the $D_{s 1}^{+}$rest frame with respect to the direction opposite to the $e^{+} e^{-}$center of mass momentum (boost direction). We define $\alpha$ as the angle between the boost direction and the $K_{S}^{0}$ momentum and $\beta$ as the angle between the $D_{s 1}^{+}$decay plane and the plane formed by the $K_{S}^{0}$ and the boost direction. The third angle $\gamma$ is defined in the $D^{*+}$ rest frame between $\pi^{+}$and $K_{S}^{0}$. The $D_{s 1}(2536)^{+} \rightarrow D^{*+} K_{S}^{0}$ decay angular distributions are shown in Fig. 2 (right). The $\cos \gamma$ distribution was fitted to the form $1+A \cos ^{2} \gamma$, and the fit yields the parameter $A=-0.70 \pm 0.03$. This measurement constrains the relative fraction $R=\Gamma_{S} /\left(\Gamma_{S}+\Gamma_{D}\right)$ 
of the $\mathrm{S}$ wave component to the range $0.277<R<0.955$, independently of the relative phase between $\mathrm{S}$ and $\mathrm{D}$ waves.

\section{Measurements of $D^{0} \rightarrow \pi^{-} l^{+} v$ and $D^{0} \rightarrow K^{-} l^{+} v$ decays}

A novel global reconstruction method is used to study the $D^{0} \rightarrow \pi^{-} l^{+} v$ and $D^{0} \rightarrow K^{-} l^{+} v$ decays [12]. Events are reconstructed assuming the process $e^{+} e^{-} \rightarrow D_{\text {tag }}^{(*)} D_{\text {sig }}^{*} X$, where $\mathrm{X}$ denotes additional $\pi^{0}, \pi^{ \pm}$and $K^{ \pm}$mesons. The tag-side $D^{ \pm(0)}$ meson is fully reconstructed in $K^{ \pm} n \pi^{ \pm(0)}$ final states with $n=1,2,3$. The signal side semileptonic decay $D^{0} \rightarrow \pi^{-}\left(K^{-}\right) e^{+}\left(\mu^{+}\right) \nu$ is studied using a missing-mass method. This method enables a very good resolution in neutrino momentum and the momentum transfer $q^{2}=\left(p_{l}+p_{v}\right)^{2}$, the accuracy $\sigma_{q^{2}} \approx 0.015 \mathrm{GeV}^{2} / \mathrm{c}^{4}$ can be achieved. This study provides an accurate information about the decay form factor $f_{D}\left(q^{2}\right)$. Additionally the decay channel $D^{0} \rightarrow K^{-} l^{+} v$ with a lower background and higher statistics was also investigated.

The relative branching fractions $\mathscr{B}\left(D^{0} \rightarrow \pi^{-} e^{+} v\right) / \mathscr{B}\left(D^{0} \rightarrow K^{-} e^{+} v\right)=0.0809 \pm 0.0080 \pm$ 0.0032 and $\mathscr{B}\left(D^{0} \rightarrow \pi^{-} \mu^{+} v\right) / \mathscr{B}\left(D^{0} \rightarrow K^{-} \mu^{+} v\right)=0.0677 \pm 0.0078 \pm 0.0047$ are obtained in a good agreement with expectations. The normalized measured $q^{2}$ distribution was fitted to different models of form factors and some deviations from predictions of simple pole [13] and ISGW2 model [14] are observed.

\section{References}

[1] K. Abe et al. (Belle Collab.), hep-ex/0507064.

[2] A. Drutskoy et al. (Belle Collab.), Phys. Rev. Lett. 94, 061802 (2005).

[3] D. Du, L. Guo, D.-X. Zhang, Phys. Lett. B406, 110 (1997).

[4] C.D. Lu, hep-ph/0305061.

[5] C.-K. Chua, W.-S. Hou, K.-C. Yang, Phys. Rev. D65, 096007 (2002).

[6] B. Blok, M. Gronau, J.L. Rosner, Phys. Rev. Lett. 78, 3999 (1997).

[7] M. Oreglia, Ph.D. thesis, Stanford University, Report No. SLAC-236 (1980).

[8] C.-H. Chen, H.-n Li, Phys. Rev. D69, 054002 (2004).

[9] V. Balagura et al. (Belle Collab.), hep-ex/0507030.

[10] N. Isgur and M. Wise, Phys. Rev. Lett. 66, 1130 (1991); M. Lu, M. Wise and N. Isgur, Phys. Rev. D45, 1553 (1992).

[11] P. Avery et al. (CLEO Collab.), Phys. Lett. B331, 236 (1994).

[12] K. Abe et al. (Belle Collab.), hep-ex/0510003.

[13] G. Armoros, S. Noguera, J. Portoles, Eur. Phys. J. C27, 243 (2003).

[14] N. Isgur and D. Scora, Phys. Rev. D52, 2783 (1995). 